\title{
ANISOTROPIA MAGNÉTICA DE COMPÓSITOS DE FERRITA DE MANGANÊS E PARAFINA*
}

\author{
Gabriel Burlandy Mota de Melo ${ }^{1}$ \\ Letícia dos Santos Aguilera ${ }^{2}$ \\ André Ben-Hur da Silva Figueiredo ${ }^{3}$ \\ Ronaldo Sérgio de Biasi ${ }^{4}$
}

\begin{abstract}
Resumo
Nanopartículas de ferrita de manganês $\left(\mathrm{MnFe}_{2} \mathrm{O}_{4}\right)$ foram dispersas em parafina e as propriedades físicas do compósito resultante foram investigadas por ressonância ferromagnética (RFM). Os resultados mostraram que a dispersão em quantidades moderadas de parafina aumenta significativamente a anisotropia magnética das nanopartículas de ferrita, um resultado que pode ser de interesse para aplicações práticas do compósito.
\end{abstract}

Palavras-chave: Ressonância ferromagética, ferrita de manganês, parafina.

\section{MAGNETIC ANISOTROPY OF MANGANESE FERRITE-PARAFFIN COMPOSITES}

\begin{abstract}
Manganese ferrite $\left(\mathrm{MnFe}_{2} \mathrm{O}_{4}\right)$ nanoparticles were dispersed in paraffin and the physical properties of this composite were investigated by ferromagnetic resonance (FMR). The results showed that the magnetic anisotropy of the ferrite nanoparticles was significantly increased by dispersion in moderate amounts of paraffin, a result that could be of interest for practical applications.
\end{abstract}

Keywords: Ferromagnetic ressonance, manganese ferrite, paraffin wax.

1 Licenciado em Física e Mestre em Ciências dos Materiais, Estudante de doutorado, Seção de Engenharia Mecânica e de Materiais, Instituto Militar de Engenharia, 22290-270 Rio de Janeiro, RJ, Brasil.

2 Bacharel em Engenharia de Petróleo, Mestre em Ciências dos Materiais Estudante de doutorado, Seção de Engenharia Mecânica e de Materiais, Instituto Militar de Engenharia, 22290-270 Rio de Janeiro, RJ, Brasil.

3 Bacharel em Física, Mestre em Engenharia Nuclear, Doutor em Ciências dos Materiais, Professor, Seção de Engenharia Mecânica e de Materiais, Instituto Militar de Engenharia, 22290-270 Rio de Janeiro, RJ, Brasil

4 Bacharel em Engenharia Elétrica, Mestre em Engenharia Elétrica e Ph. D. em Engenharia Elétrica, Professor Emérito, Seção de Engenharia Mecânica e de Materiais, Instituto Militar de Engenharia, 22290-270 Rio de Janeiro, RJ, Brasil. 


\section{INTRODUÇÃO}

A ferrita de manganês em forma nanométrica é um material muito investigado para aplicações práticas por apresentar propriedades como alta magnetização de saturação e boa estabilidade química [1]. Entretanto, devido à atração magnética, as nanopartículas de ferrita tendem a formar aglomerados que podem comprometer seu desempenho em certas aplicações. Uma das soluções propostas tem sido dispersar as nanopartículas em materiais não magnéticos [1-10]. Um material não magnético conveniente é a parafina, um material relativamente barato e com um baixo ponto de fusão.

O objetivo deste trabalho foi investigar a influência da dispersão em parafina na anisotropia magnética da ferrita de manganês. Para isso, foram fabricadas amostras com diferentes diluições de ferrita em parafina.

\section{MATERIAIS E MÉTODOS}

\subsection{Preparação das Amostras}

Nanopartículas de ferrita de manganês foram sintetizadas pelo método de combustão [11]. Quantidades estequiométricas de nitrato de manganês, $\mathrm{Mn}(\mathrm{NO} 3) 2.4 \mathrm{H} 2 \mathrm{O}$, Aldrich, 98\%, e nitrato de ferro, $\mathrm{Fe}(\mathrm{NO} 3) 3.9 \mathrm{H} 2 \mathrm{O}$, Aldrich, 98\%, foram dissolvidas em água destilada para obter a solução precursora. Em seguida, foi adicionada uma solução de glicina, $\mathrm{C} 2 \mathrm{H} 5 \mathrm{NO} 2$, Aldrich, 98.5\%, com uma relação glicina-nitratos $(\mathrm{G} / \mathrm{N})$ de 0,5 . A solução resultante foi agitada por $20 \mathrm{~min}$ à temperatura ambiente e aquecida a $100{ }^{\circ} \mathrm{C}$ para iniciar a autocombustão. $\mathrm{O}$ pó resultante foi disperse em parafina fundida com razões em peso parafina/ferrita (P/F) de $0,5,10,15$ e 20, usando um banho ultrassônico de $40 \mathrm{kHz}$.

\subsection{Medidas de Difração de Raios $X$}

O difratograma de raios $X$ foi registrado em um difratômetro PANalytical X'Pert PRO usando radiação $\mathrm{K} \alpha$ do $\mathrm{Cu}(1.5418 \AA$ A), e ajustado pelo método de Rietveld, com auxílio do software High Score 3.0.

\subsection{Espectros de FMR}

As medidas de ressonância ferromagnética foram executadas à temperatura ambiente e $9,50 \mathrm{GHz}$ em um espectrômetro Varian $\mathrm{E}-12$, com uma frequência de modulação do campo magnético de $100 \mathrm{kHz}$. A potência de Microondas foi $5 \mathrm{~mW}$ e a amplitude do campo de modulação foi $1 \mathrm{mT}$. O campo magnético foi calibrado com um gaussímetro de RMN.

Os resultados experimentais foram ajustados aos espectros teóricos usando um programa de computador desenvolvido por Taylor e Bray [12] para espectros de ressonância paramagnética e adaptado por Griscom [13] para espectros de ressonância ferromagnética. 


\section{RESULTADOS E DISCUSSÃO}

A figura 1 mostra o difratograma do pó antes da dispersão. $O$ refinamento de Rietveld revelou que o material contém 84,3\% de ferrita de manganês (MnFe2O4), com um tamanho médio de cristalito de $7 \mathrm{~nm}$, uma constante de rede de $0,85 \mathrm{~nm}$ e um goodness of fit (GOF) de 1,47. O material também contém $15,6 \%$ de hematita (Fe2O3) e $0,1 \%$ de goetita, $\mathrm{FeO}(\mathrm{OH})$.

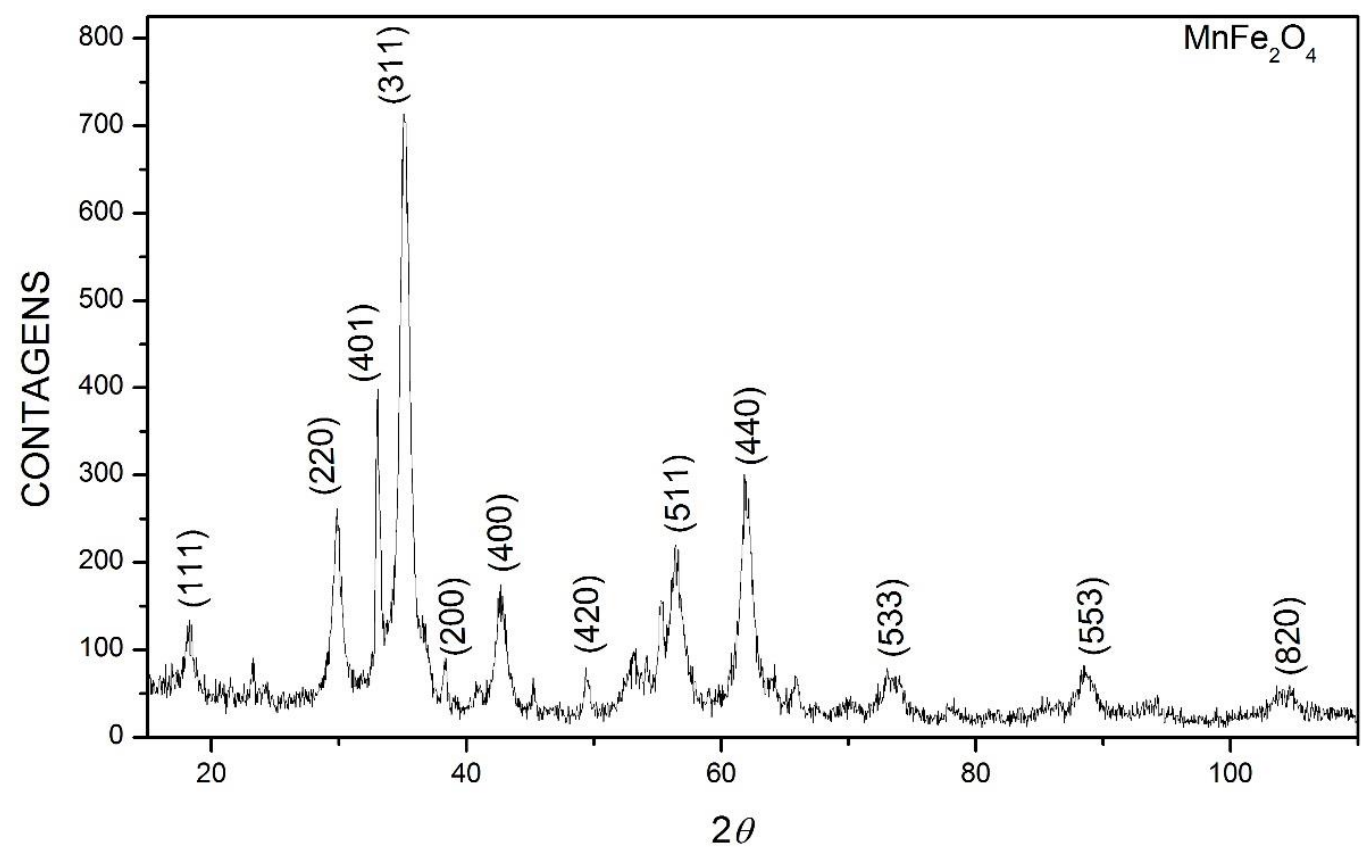

Figura 1. Difratograma do pó de ferrita.

Os picos rotulados como (111), (022), (311), (400), (511), (440), (533) e (553) na Fig. 1 são atribuídos à ferrita de manganês, enquanto os picos (401) e (420) são atribuídos à hematita e os picos (200) e (820) são atribuídos à goetita.

De acordo com os resultados experimentais mostrados na Tabela 1 e na figura 3 , a dispersão em quantidades moderadas de parafina aumenta significativamente o campo de anisotropia das nanopartículas, um resultado que pode ser de interesse para aplicações práticas. Este comportamento pode se dever ao efeito da parafina sobre a constante de rede da ferrita ou sobre a desordem dos spins na superfície das nanopartículas [1]. A diminuição da anisotropia magnética para altos valores de P/F é atribuída a uma diminuição do campo local que age sobre as partículas, o que faz com que o comportamento superparamagnético se estenda além da parte de baixo campo do espectro, como é observado na ferrita de magnésio [15].

Um espectro típico, obtido em uma amostra com uma razão $P / F$ de 5 , é mostrado na figura 2. 


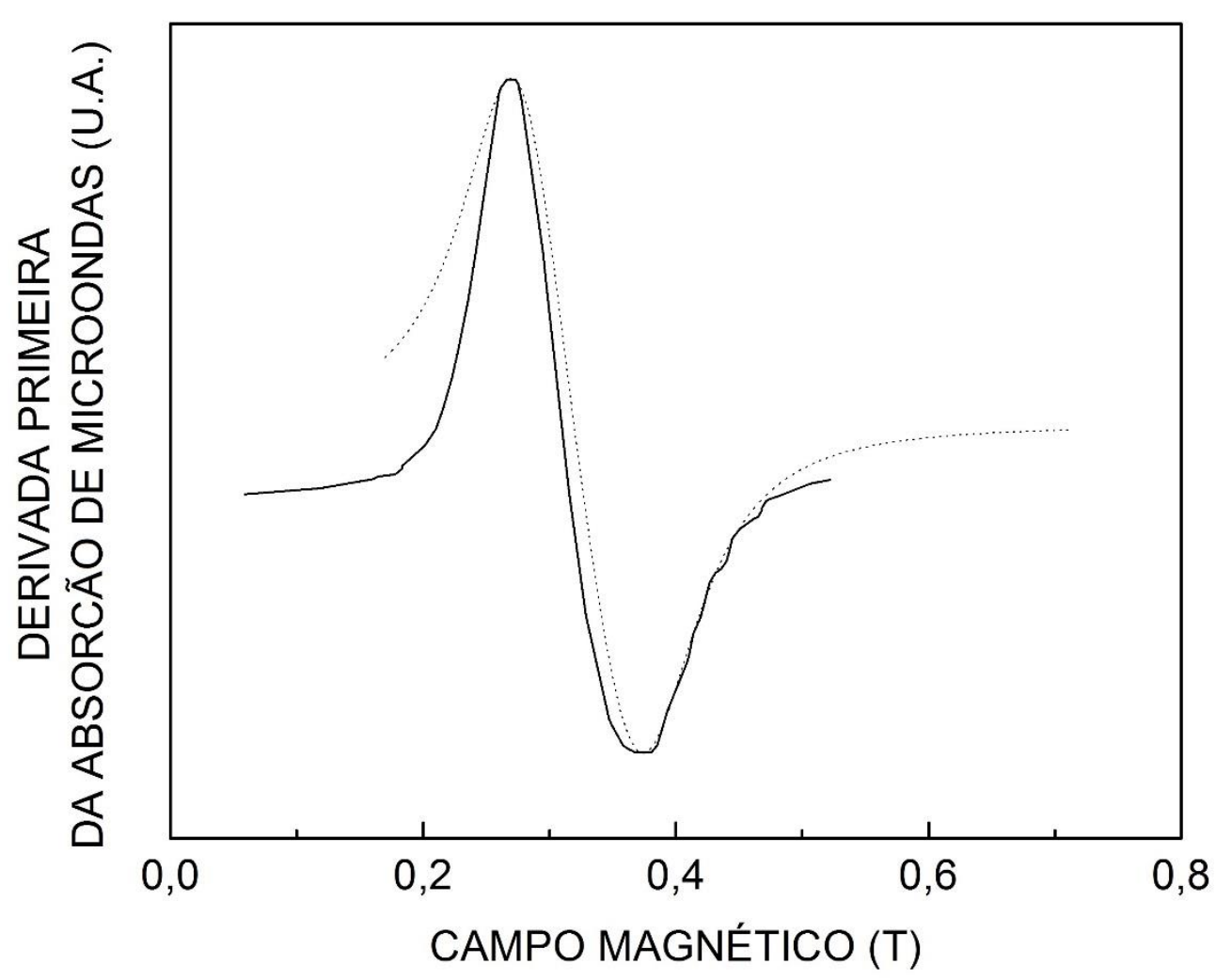

Figura 2. Ressonância ferromagnética do compósito parafina ferrita 5 (P/F 5).

O ajuste foi razoável para todas as amostras, exceto na região de baixos campos, na qual a inclinação da curva de amplitude foi muito mais nas curvas experimentais do que nas curvas teóricas. A diferença é atribuída [14] ao efeito do superparamagnetismo. Os parâmetros dos ajustes são mostrados na Tabela 1.

Tabela 1. Parâmetros usados nas simulações dos espectros de ressonância magnética.

\begin{tabular}{cccc}
\hline $\mathbf{P} / \mathbf{F}$ & fator $\boldsymbol{g}$ & $\begin{array}{c}\text { Campo de anisotropia, } \\
\boldsymbol{H}_{\boldsymbol{a}}(\mathbf{m} \mathbf{)})\end{array}$ & $\begin{array}{c}\text { Largura de linha } \\
\text { intrínseca, } \boldsymbol{\sigma}(\mathbf{m T})\end{array}$ \\
\hline 0 & 2,10 & $-29,0$ & 78,5 \\
\hline 5 & 2,10 & $-41,5$ & 90,3 \\
\hline 10 & 2,10 & $-47,0$ & 103,3 \\
\hline 15 & 2,10 & $-42,0$ & 97,9 \\
\hline 20 & 2,10 & $-37,0$ & 86,8 \\
\hline
\end{tabular}

A Figura 3 mostra a variação o campo de anisotropia Ha com a razão $P / F$. $O$ campo de anisotropia passa por um máximo em $\mathrm{P} / \mathrm{F}=10$, pois o sinal negativo indica apenas que a direção de fácil magnetização da célula unitária da ferrita é a direção [111]. 


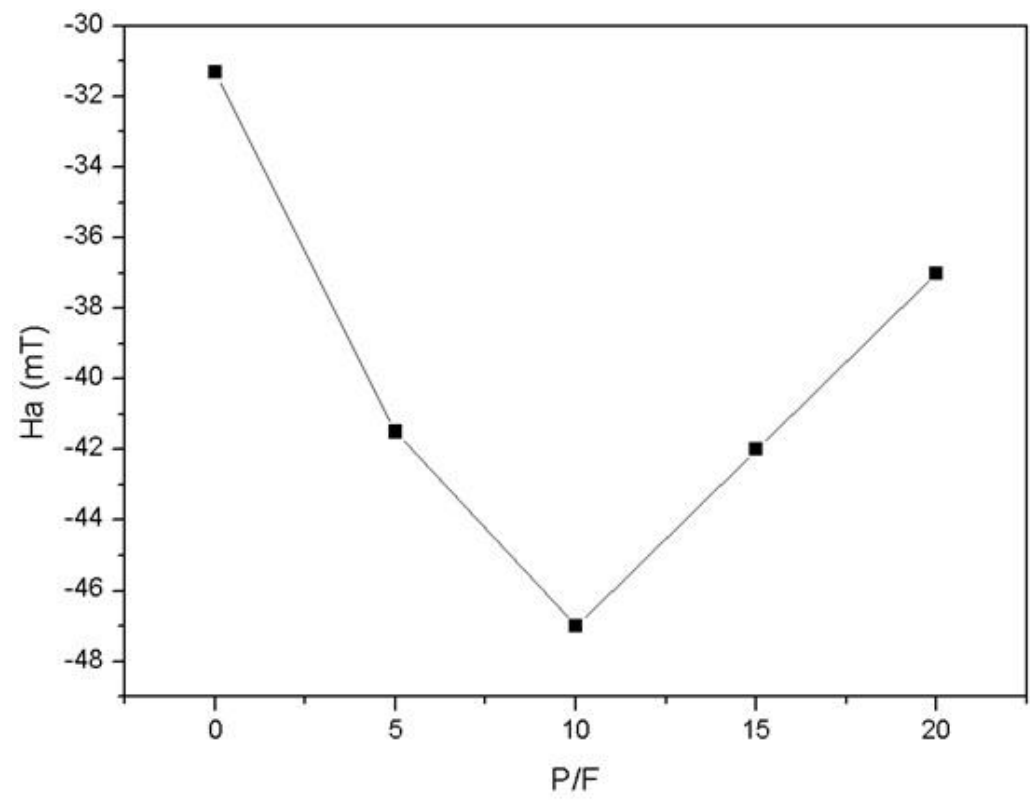

Figura 3. Comportamento do campo de anisotropia em função da razão $P / F$. (As linhas retas são apenas para guiar os olhos).

De acordo com os resultados experimentais mostrados na tabela 1 e na figura 3 , a dispersão em quantidades moderadas de parafina aumenta significativamente 0 campo de anisotropia das nanopartículas, um resultado que pode ser de interesse para aplicações práticas. Este comportamento pode se dever ao efeito da parafina sobre a constante de rede da ferrita ou sobre a desordem dos spins na superfície das nanopartículas [1]. A diminuição da anisotropia magnética para altos valores de P/F é atribuída a uma diminuição do campo local que age sobre as partículas, o que faz com que o comportamento superparamagnético se estenda além da parte de baixo campo do espectro, como é observado na ferrita de magnésio [15].

\section{CONCLUSÃO}

A diluição da ferrita na parafina provoca variação do campo de anisotropia magnética $\left(\mathrm{H}_{\mathrm{a}}\right)$, com comportamento decrescente até $\mathrm{P} / \mathrm{F} 10$, onde atinge um ponto de mínimo e a partir deste valor um comportamento crescente, tendendo a retornar ao valor de $\mathrm{H}_{\mathrm{a}}$ sem parafina (P/F 0), sugerindo que a parafina diminui a vibração da rede cristalina da ferrita, permitindo um melhor alinhamento dos spins catiônicos, sendo mais efetiva em P/F 10.

\section{Agradecimentos}

Os autores agradecem à CAPES e ao CNPq pelo apoio financeiro.

\section{REFERÊNCIAS}

1 Aslibeiki B, Kameli $\mathrm{P}$. Magnetic properties of $\mathrm{MnFe2O4}$ nano-aggregates dispersed in paraffin wax. Journal of Magnestism and Magnetic Materials. 2015; 385: 308-312.

2 Stejskal J, Trchová M, Brodinová J, Kalenda P, Fedorova SV, Prokeš J, Zemek J. Coating of zinc ferrite particles with a conducting polymer, polyaniline. Journal of Colloid Interface Science. 2006; 298: 87-93. 
3 Li BW, Shen Y, Yue ZX, Nan CW. Influence of particle size on electromagnetic behavior and microwave absorption properties of Z-type Ba-ferrite/polymer composites. Journal of Magnetism and Magnetic Materials. 2007; 313: 322-328.

4 Dosoudil R, Usáková M, Franek J, Grusková A, Sláma J. Dispersion of complex permeability and EM-wave absorbing characteristics of polymer-based composites with dual ferrite filler. Journal of Magnetism and Magnetic Materials. 2008; 320: e849-e852

5 K. Shimba N, Tezuka S. Sugimoto. Magnetic and microwave absorption properties of polymer composites with amorphous $\mathrm{Fe}-\mathrm{B} / \mathrm{Ni}-\mathrm{Zn}$ ferrite nanoparticles. Materials Science and Engineering B. 2012; 177: 251-256.

6 Malana MA, Qureshi RB, Ashiq MN. Adsorption studies of arsenic on nano aluminium doped manganese copper ferrite polymer (MA, VA, AA) composite: Kinetics and mechanism. Chemical Engineering Journal. 2011; 172: 721-727.

7 Bayrakdar $\mathrm{H}$. Complex permittivity, complex permeability and microwave absorption properties of ferrite-paraffin polymer composites. Journal of Magnetism and Magnetic Materials. 2011; 323: 1882-1855.

8 Hayashi K, Maeda K, Moriya M, Sakamoto W, Yogo T. In situ synthesis of cobalt ferrite nanoparticle/polymer hybrid from a mixed $\mathrm{Fe}-\mathrm{Co}$ methacrylate for magnetic hyperthermia. Journal of Magnetism and Magnetic Materials. 2012; 324: 3158-3164.

9 Borah S, Bhattacharyya NS. Broadband magneto-dielectric response of particulate ferrite polymer composite at microwave frequencies. Composites Part B. 2012; 43: 1988-1994.

10 Tong SY, Tung MJ, Ko WS, Huang YT, Wang YP, Wang LC, Wu JM. Effect of Ni fillers on microwave absorption and effective permeability of $\mathrm{NiCuZn}$ ferrite/Ni/polymer functional composites. Journal of Alloys and Compounds. 2013; 550: 39-45.

11 Aruna ST, Mukasyan AS. Combustion synthesis and nanomaterials. Current Opinion in Solid State and Materials Science. 2008; 12: 44-50.

12 Taylor PC, Bray PJ. Computer Simulations of magnetic resonance spectra observed in polycrystalline and glassy samples. Journal of Magnetic Resonance. 1970; 2: 305-331.

13 Griscom DL. Ferromagnetic resonance of precipitated phases in natural glasses. Journal of Non-Crystalline Solids. 1984; 67: 81-118.

14 de Biasi RS, Lopes RDS. Magnetocrystalline anisotropy of NiCoFe2O4 nanoparticles. Ceramics International. 2016; 42: 9315-9318.

15 de Biasi RS, Devezas TC, Anisotropy field of small magnetic particles as measured by resonance. Journal of Applied Physics. 1978; 49: 2466-2469.. 